\title{
LAS INFLEXIONES DE INICIO Y TÉRMINO DEL CICLO DE EXPANSIÓN DEL SALITRE (1872-1919). UNA CRÍTICA AL NACIONALISMO METODOLÓGICO*
}

\author{
INFLECTIONS OF THE BEGINNING AND END OF THE NITRATE EXPANSION \\ CYCLE (1872-1919). A CRITIQUE OF METHODOLOGICAL NATIONALISM
}

\author{
Sergio González Miranda**
}

\begin{abstract}
El ciclo de expansión del salitre suele enmarcarse entre 1880 y 1930, sin embargo, no existen suficientes argumentos, más allá de la incorporación en 1880 de Tarapacá, la principal provincia salitrera, al Estado-Nacional y que, en 1930, la crisis de la Gran Depresión tuvo un impacto profundo en esta economía.

Aquí, a partir de un análisis de la propia industria salitrera, se proponen otros momentos de inflexión para el despegue definitivo de la economía salitrera y para su término, los que serían 1872 y 1919, respectivamente. También se propone una crítica al nacionalismo metodológico que ha imperado en las perspectivas peruana y chilena en el estudio de la economía y sociedad del salitre.

Palabras claves: Ciclo de expansión del salitre, inflexión económica, nacionalismo metodológico.
\end{abstract}

The nitrate expansion cycle is usually placed between 1880 and 1930, however, there are not enough arguments, beyond the incorporation in 1880 of Tarapacá, the main nitrate province, to the nation-state and that, in 1930, the Great Depression had a deep impact on this economy.

Here, since an analysis of the nitrate industry itself, other moments of inflection are proposed for the nitrate economy to leave definitely and for its end that would be 1872 and 1919, respectively. A critique of methodological nationalism is also proposed that has prevailed in the Peruvian and Chilean perspectives in the study of nitrate economy and society.

Key words: nitrate expansion cycle, economic inflection, methodological nationalism.

\section{Introducción}

Enmarcar el ciclo del salitre en su fase expansiva resulta relevante no solo en su dimensión económica, saber cuándo esta economía inicia su despegue definitivo y cuándo declina, sino también en sus dimensiones política y social, quiénes fueron los que le dieron ese impulso y quiénes enfrentaron la decadencia. Cuáles fueron las administraciones estatales y las políticas salitreras que imperaban en dichos momentos. Cuál fue la sociedad que enfrentó el desafío de trabajar en esta industria durante el auge y la crisis.

El caso específico de esta industria nos plantea un problema peculiar: surgió en un territorio bajo la soberanía de un país, Perú, y concluyó bajo la soberanía de otro, Chile. Por lo tanto, nos enfrentamos a enfoques e intereses distintos al momento de establecer el marco temporal en que se desenvolvió esta actividad. Para el Perú el periodo relevante podría ser aquel de los gobiernos de José Balta, Manuel Pardo y Mariano Ignacio Prado y, para Chile, los gobiernos entre Aníbal Pinto y Arturo Alessandri. Para el fisco y sus impuestos, por cierto, el salitre es visto desde una perspectiva nacional, por ende, los análisis económicos en uno y otro país destacan lo que les corresponde de esta industria, para el Perú desde que se comenzó a aplicar un impuesto al salitre: el 30 de noviembre de 1868 (Noejovich y Vento 2009:9) y, para Chile, puede ser el impuesto que estableció por ley el 12 de septiembre de 1879 .

Lo anterior nos enfrenta con lo que algunos autores, generalmente venidos desde los estudios migratorios, definen como "nacionalismo metodológico", que sería "una versión clásica de observación de la realidad que presupone al Estado-Nación como principio organizador de la sociedad" (Morcillo 2011:759), o como una perspectiva a escala del Estado-Nación que se considera un enfoque "aparentemente neutral y automático de las reflexiones

\footnotetext{
* Resultado Proyecto Fondecyt No 1130517

** Universidad Arturo Prat, Instituto de Estudios Internacionales (INTE), Iquique, Chile. Correo electrónico: sgonzale@ unap.cl
} 
teóricas" (Masso 2013:73). Por ello, para el Perú el ciclo del salitre tuvo relevancia cuándo reemplazó al guano como un flujo financiero para pagar la deuda externa y los gastos públicos e, igualmente, para Chile el ciclo del nitrato se inicia en 1880, cuándo ocupa y administra Tarapacá, y concluye cuándo ya los aportes por salitre y yodo eran escasos, en la década de 1930.

El estudio de las sociedades contemporáneas, específicamente en el periodo republicano, corre el riesgo de superponer los requerimientos del EstadoNación y sus fronteras con los problemas propios de la ciencia social. Los límites de la ciencia social, entre ellas la Historia, no tienen necesariamente que coincidir con los de un país, como sucede con el nacionalismo metodológico.

El nacionalismo metodológico es una perspectiva que se superpone a otras, como la regional o la local, desde las cuales es posible superar la barrera de las fronteras nacionales, como es el caso del estudio de regiones transfronterizas (Jessop 2004) o de localidades que habitan las fronteras (Grimson 2005). En este caso, vemos a una región (Tarapacá) -donde se desarrolló la industria del salitre- que estuvo bajo la administración y soberanía de dos Estados nacionales: Perú y Chile. La inflexión de término de dicha industria no puede ser el momento en que el primer Estado-nacional dejó de administrar ese territorio, como tampoco la de inicio cuándo el segundo Estado-nacional se hizo cargo de esa administración. Las inflexiones de inicio y término del ciclo de expansión del salitre dependen de la propia industria y su devenir, a escalas regional y mundial.

Este artículo aborda las inflexiones de inicio y término del ciclo de expansión del salitre, analizadas desde su propio desenvolvimiento como industria.

\section{El inicio de la expansión del salitre: la inflexión de 1872}

La economía del nitrato se inicia en la provincia de Tarapacá durante el primer tercio del siglo diecinueve como ya lo comprobaron los investigadores clásicos de la Historia del Salitre (Hernández 1930; Bermúdez 1963), pero no podemos hablar ni de una economía estable y menos en expansión, tampoco de una sociedad en (y menos del) desierto asociada a esta actividad. El censo de 1841 registró una población de 3.758 habitantes, y aquella relacionada a la actividad minera solamente estaba en los asentamientos argentíferos de Santa Rosa (10,6\%) y Huantajaya $(5,7 \%)$, mientras la categoría "salitrera" tiene $0,1 \%$. El sistema de lixiviación conocido como "paradas" obligaba a moverse de un manto a otro en poco tiempo, por lo tanto los campamentos eran precarios y temporales. Recién con la aparición del sistema de lixiviación del salitre por vapor se puede comenzar a identificar una industria del nitrato y una sociedad con campamentos y pueblos más estables. También las caletas que exportaron tempranamente salitre desaparecieron o se transformaron en puertos menores y mayores. Entre las primeras tenemos a caleta Molle, entre las segundas a Junín y Pisagua.

Durante el periodo de 1830 a 1834 se exportaron 361.386 quintales españoles de salitre, cuándo esta economía estaba lejos de ser una industria, pues su producción se sustentaba en una tecnología móvil con un sistema de lixiviación muy simple a fuego directo que beneficiaba caliches de alta ley. Ya con un notorio mejoramiento de la tecnología salitrera (Crozier 1997), durante el periodo de 1850 a 1854 la exportación alcanzó 3.260.492 quintales españoles de nitrato de soda y en el quinquenio de 1860 a 1864 aumentó a más del doble: 6.979.208, lo que podría calificarse de una expansión; empero, en la década siguiente solamente en 1875 se superó esa cifra llegando a 7.943.000, recién en 1885 se superaría esa cifra (Semper y Michels 1908:134). Desde un punto de vista técnico, el ingeniero Belisario Díaz Ossa, profesor de la cátedra Salitre de la Universidad de Chile en la década de 1920, afirma: "la industrialización propiamente tal comenzó solo en 1870, ensayando numerosos aparatos de lixiviación metódica..." (1926:156), lo que implica sin duda una inflexión tecnológica en esta industria.

El censo de 1866 (Ruz et al. 2008) registra un cambio relevante, no solo señala que la población de Tarapacá de 1841 aumentó casi dos veces (9.646 habitantes), sino el notorio aumento se concentró en el puerto de Iquique $(33,7 \%$ ) y en los incipientes pueblos salitreros $(5,2 \%)$. A fines de esa década este puerto ya contaba con una Aduana, iniciativa del gobierno de José Balta, en vista del aumento de la producción de nitrato de soda. Como bien lo dicen Noejovich y Vento: "el salitre tuvo un régimen diferente (al guano); hasta 1856 la explotación era libre y recién el 30 de noviembre de 1868 es estableció un impuesto de 4 centavos (2009:9). 
De todas formas, para esa época todavía la economía del guano era la principal riqueza del Perú y el salitre una incertidumbre. El puerto del Callao era el que concentraba las operaciones del guano, mientras Valparaíso comenzaba a transformase en la plataforma comercial del nitrato que, por esa razón, será conocido en el mercado europeo como nitrato de Chile. Según Manuel Fernández Canque, "el salitre se había transformado ya en una mercancía internacional a comienzos de la década de 1830 . La producción a gran escala, sin embargo, comenzó en la década de 1860 y continuó su incremento en relación inversa a la declinación de la producción del guano" (1988:4). ¿Cuál fecha podríamos señalar como el punto de inflexión de la expansión del salitre?

Se observa un notorio descenso de las exportaciones durante el gobierno de José Balta, durante la segunda mitad de la década de 1860 (Gráfico 2), y un punto de arranque al iniciarse la década siguiente, llegando a su mayor expresión en 1875, cuándo en Tarapacá comenzó un boom salitrero con la construcción de máquinas como lo veremos más adelante.

Como se ha señalado, el salitre comenzó a ser un fertilizante comercializable en el mercado internacional en la década de 1830, pero su despegue fue cuatro décadas después al alcanzar y superar al guano en el marco de las exportaciones peruanas. Noejovich y Vento son más específicos al afirmar que "podemos asumir una estrategia global, probablemente no exteriorizada abiertamente, hacia un incremento del salitre como producto de exportación, en la medida que el recurso guanero quedó comprometido con el Contrato Dreyfus (2009:10). En otras palabras, el éxito de la economía salitrera no fue independiente de la política económica peruana, posiblemente la libertad de explotación hasta 1868 fue clave. Por esta razón, los salitreros tarapaqueños se resistieron al cambio de política que propuso el presidente Manuel Pardo en 1873 con el estanco salitrero.

Como bien señala Heraclio Bonilla, el salitre "fue un fertilizante que desde 1870 empezó a desplazar al guano en el mercado internacional" (1985:15). Perú estaba desde las décadas anteriores recurriendo a créditos externos para financiar sus obras de infraestructura, como ferrocarriles, y el gasto público, sobre la base de sus recursos naturales, específicamente el guano. Hacia 1872, cuándo asume la presidencia Manuel Pardo, quien además es el primero con un proyecto político de desarrollo nacional bien definido, encuentra que la economía del guano no solo estaba destinada íntegramente a servir la deuda externa, sino que dejaba un déficit, por lo tanto comienza a enfocar su interés en el salitre. El Gráfico 1 nos demuestra que en 1872 el salitre aporta al fisco peruano un porcentaje muy parecido al del guano, para superarlo al año siguiente. La crisis financiera del Estado peruano introdujo a la economía salitrera en el mismo problema del gasto fiscal que había asumido la economía del guano.

Según Miller y Greenhill, el impuesto que se aplicó a las exportaciones de salitre bajo el gobierno de Balta, "de 4 centavos el quintal ( 3 chelines y 2 peniques la tonelada), fijado en 1868 , en modo alguno era opresivo, porque el precio del salitre fluctuaba entre $£ 11$ y $£ 15$ la tonelada, y los costes estaban cayendo" (2011:38), pero no resolvía las necesidades de financiamiento del Estado peruano. Además, señalan estos autores, "los peruanos achacaban la caída en las ventas del guano, que amenazaba la principal fuente de rentas del gobierno, a la extensa producción de salitre barato, lo que animó todavía más a Lima a examinar la industria" (2011:39). Sería más preciso hablar de Lima que de los peruanos en general respecto de la mirada crítica a la venta de salitre, ya que eran también peruanos, pero tarapaqueños, los salitreros que habían puesto a la economía del guano en situación incómoda en el mercado de los fertilizantes. Fue "el círculo de Lima", como lo denominó Billinghurst (1875), un grupo asociado a la banca peruana, el más interesado en intervenir a la industria salitrera, en alianza con grandes empresas extranjeras, específicamente con la Casa Gibbs (Ravest 2008, Miller y Greenhill 2011).

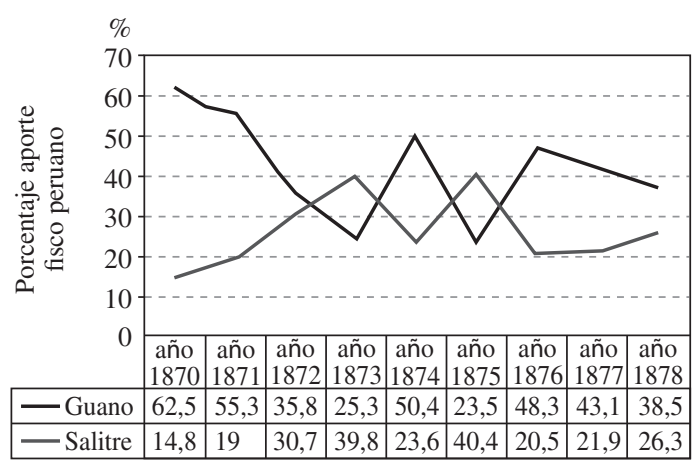

Gráfico 1. Aporte al fisco peruano del guano y del salitre 1870-1878.

Fuente: Carlos Contreras 2012: 378. 
Sorprende leer de especialistas británicos como Miller y Greenhill señalar que Tarapacá, "una región escasamente poblada e infértil, la industria del salitre se hallaba aislada económica y físicamente del resto del Perú"(...) "Los salitreros habían creado una economía de enclave en Tarapacá. Su mercancía exportadora de rápido crecimiento, despachada desde Iquique, afectaba a un sector limitado pero dejaba sin tocar al resto del país" (2011:42). Precisamente este es el más clásico enfoque a escala nacional, donde no es posible entender que desde una "región marginal" se pueda desarrollar una actividad económica dominante sin la participación del centro político y económico nacional. ¿Hubiese sido posible un desarrollo de economía salitrera sin la intervención del Estado-Nación? Aquí es relevante seguir a Wallerstein, quien hace una crítica a la perspectiva que privilegia al Estado-Nación y sus fronteras como unidad de análisis, subestimando "las relaciones de explotación y dominación del sistema-mundo en las que el desarrollo y subdesarrollo son las dos caras de la misma moneda" (Grosfoguel 2006:45). Y así aconteció en la economía del salitre bajo la política chilena después de 1884, cuándo los empresarios salitreros, mayoritariamente extranjeros, se organizaron para el control del comercio del salitre y el yodo. De hecho en ese periodo, y hasta 1910, la presencia de capitales chilenos y peruanos en la industria salitrera de Tarapacá fue marginal.

La visión Estado-céntrica llevó, durante la economía del guano, también a una actividad regional, a crear los bancos La Providencia, Nacional del Perú, Lima y Bancos Hipotecarios (Reyes 1979:103), algunos de esos bancos operaron después en la economía del salitre. Sin embargo, igualmente el gobierno peruano debió recurrir, para una mejor gestión en el mercado internacional, a empresas extranjeras como la casa Gibbs. El poder estratégico que la casa Gibbs alcanzó con el gobierno peruano en la temprana economía del salitre se basó en su papel en la economía del guano (Mathew 2099).

Thomas O'Brien nos aclara el momento en que, frente al estancamiento de la economía del guano, los círculos limeños comenzaron a observar hacia el margen sur del país: "A comienzos de los sesentas los intereses de este grupo se centraron en las empresas financieras y comerciales de Lima. Una parte de los recursos capitales se invirtieron rápidamente en ampliar los trabajos salitreros en Tarapacá. Entre 1870 y 1874 seis empresas con un capital nominal de 3.620 .000 soles fue tomando forma en Lima para producir salitre en Tarapacá. A fines de 1872 el recién formado Banco Nacional del Perú fundó una sede en Iquique y, en los siguientes dos años, extendió 4 millones de soles en créditos a productores de salitre" (1985:15).

La economía del salitre es vista como "enclave" por tratarse de una actividad minera que se ubica en una región aislada, escasamente poblada y alejada del centro político, pero sobre todo por la desconfianza que desde lugares con esas "características" pueda surgir una sociedad con densidad cultural y capacidad endógena de desarrollo (Stöhr 1992).

Si nos enfocáramos en los intereses del EstadoNación peruano, de los banqueros limeños y de las casas comerciales extranjeras, sería difícil establecer el momento de auge de la economía del salitre, debido a la intervención que desde los gobiernos se hizo de esta economía, generando incertidumbre y especulación.

La perspectiva desde el nacionalismo metodológico se centraría para establecer el punto de inflexión en los impuestos y la renta fiscal, en el estanco salitrero o en la ley de expropiación, etc. Sin embargo, la pregunta es más simple: ¿cuándo el nitrato de soda se transformó en el producto dominante en el mercado internacional de los fertilizantes?

La importancia del salitre no puede ser vista solo en comparación con el guano, porque ya estaban participando otros productos azoados en el mercado de los fertilizantes, pero era en ese momento el guano el principal competidor. El guano enfrentaba hacia 1872 no solo el agotamiento de las covaderas de las islas Chinchas, sino como lo afirma O'Brien "incluso el guano de alta calidad solo tenía la mitad de nitrógeno que contenía el salitre, mejorando por lejos la petición de este último en el mercado de fertilizantes europeos" (1985:9).

Si bien el Gráfico 2 nos indica que la producción de guano siempre superó a la del salitre en la década de 1868 a 1877 , el precio del primero iba en caída mientras el del segundo en ascenso: Miller y Greenhill señalan, para ese periodo, un precio promedio para el guano de $£ 12$ por tonelada, en cambio para el salitre era de $£ 15$ por tonelada (2010:38 y sgte. Cuadros 1.1 y 1.2)

El año 1872 será por diversas razones un punto de inflexión: 1. La exportación de salitre alcanza en generación de ingresos a la del guano; 2. Con el triunfo electoral civilista de 1872 en Perú (Mücke 2004), se inicia el periodo gubernamental 


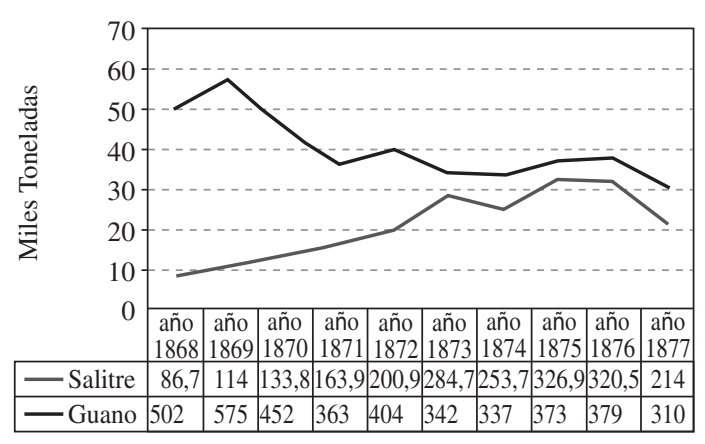

Gráfico 2. Producción de Salitre y Guano 1868 - 1877 Fuente: Miller y Greenhill 2010:38

de Manuel Pardo, quien diseñará la política salitrera más importante de la década y que marcará el ciclo del salitre, tanto respecto de la propiedad de esta industria como del modelo económico; 3. En 1872 concluye, para el Perú, el periodo de los empréstitos y de la aparente opulencia guanera, y se enfrenta una crisis que, al decir de Heraclio Bonilla, tuvo una importancia capital en "el colapso de la década de 1870" (Bonilla 1985:93), que arrastró, en definitiva, al colapso de todo el Estado-Nación peruano en esa década. Término que también utiliza Shane J. Hunt: "el colapso hacia el final de la Edad del Guano fue completo y devastador" (2011:79). Cabe destacar que no fue una crisis generada completamente en Perú, sino también respondió a lo que Carlos Marichal denomina "la primera auténtica crisis financiera mundial", "el carácter global se manifestó en el hecho de que la crisis golpeó a numerosas economías de la periferia: por ejemplo, llevó a la bancarrota, entre 1875 y 1876, a los gobiernos enormemente endeudados del Imperio Otomano - Turquía y Egipto-, así como al de Perú, el más endeudado de América Latina" (2009:24).

Desde un análisis exclusivamente económico, que no es lo que se pretende aquí, la inflexión de arranque de la industria del salitre puede ser cualquier año posterior a 1868, sin embargo, hay también una razón sociopolítica para establecerla en 1872: la capacidad de organización que tuvieron los salitreros de Tarapacá para enfrentar a la política gubernamental y parlamentaria del Perú que se expresó en la resistencia al estanco salitrero de 1873.

Si bien la expansión salitrera de la década de 1870 puede ser vista como modesta respecto de la observada en la década siguiente, bajo la política salitrera y administración chilenas generó la presencia de las Compañías inglesas y de otras nacionalidades europeas en esta industria y que resultaron clave en el periodo chileno en la organización de esta economía. Antes de 1870 el predominio era de capitales peruanos-tarapaqueños y chilenos (Billinghurst 1875). Entre 1870 y 1872 se organizaron en Lima seis compañías: Barrenechea, Esperanza, Alianza, La Peña, Providencia y Rimac, que se sumaron a las ya existentes de origen tarapaqueño, mientras los capitales chilenos, constituyeron en Valparaíso hacia 1873 diez compañías: Sacramento, Solferino, América, Chucumata, N. Carolina, Pisagua, San Carlos Negreiros, Valparaíso y California (Bravo 1990:48).

Siguiendo a Guillermo Billinghurst, se habrían construido 56 oficinas con esa tecnología entre 1872 y 1878 (1889:15), mientras al sur de río Loa solo era posible identificar a la salitrera de la Compañía de salitres y ferrocarril de Antofagasta, que explotaba los salitres del salar del Carmen. El 27 de noviembre de 1873 su antecesora, la Compañía Exploradora del Desierto, obtuvo la autorización del gobierno de Bolivia para iniciar sus faenas. Mientras las regiones salitreras del Toco y Taltal solo estaban en la fase de cateos. Tarapacá, en cambio, vivía ese boom que tuvo mucho de especulación, algunas máquinas solo tuvieron por destino la expropiación o surgieron a propósito de ella. Como bien señalan Miller y Greenhill, los salitreros "que en 1873 habían gozado de unos sustanciales retornos y resistieron vigorosamente (el estanco salitrero), ciertamente fue ahora (frente a la expropiación) bastante servicial" (2010:46), debido fundamentalmente a la caída de los precios del nitrato.

Sumado a lo anterior, Miller y Greenhill afirman que "Perú usó su monopolio del salitre como un medio para explotar a los consumidores. No advirtió que elevar su precio simplemente animaba a los intrusos a exportar rentablemente a pesar de los impuestos que acababan de ser aplicados..." (2010:54). Estos autores afirman que "la expropiación fue una innovación audaz y hasta especulativa, obligada por la fuerza de las circunstancias. Tanto el estanco -un atractivo compromiso entre el principio y la necesidad- como la expropiación no tuvieron su origen en el gobierno de Pardo, sino que emanaron del Congreso..." (2010:63). Más allá de la autoría de estas medidas que, de todos modos, sostenemos fueron pilares de la política salitrera de Pardo, tuvieron un efecto contrario a lo buscado por el gobierno peruano, afectando el 
desenvolvimiento normal de esta industria, inoculando la especulación y la incertidumbre. Aún así, no se puede negar que fue en esta década cuándo el salitre pasó al primer plano de las industrias latinoamericanas de la época. También nunca antes ni después se tendrá una innovación tecnológica en la lixiviación del nitrato de soda tan exitosa como la acontecida en la década de 1870, al reconocer que el sistema de lixiviación Shanks fue introducido entre 1876 y 1878.

En definitiva, identificamos el año 1872, por las razones ya señaladas anteriormente, como el punto de inflexión de lo que podríamos denominar el inicio de la expansión del salitre; en otras palabras, el despegue de esta industria se produjo cuatro décadas después desde su primera exportación.

\section{La inflexión de término del ciclo de expansión del salitre: 1919}

Si bien para la economía chilena es evidente que el nitrato de soda tiene relevancia estratégica a partir de 1880, es preciso reconocer a las décadas anteriores como fundamentales para entender a esta industria en toda su extensión temporal, evitando el "nacionalismo metodológico" que nos lleva a pensar el fenómeno dentro de los límites de las fronteras nacionales. En otro trabajo hemos discutido las razones que algunos especialistas tuvieron para identificar al año 1880 como el punto de inflexión del ciclo de expansión del salitre. Solo adelantaremos que no son autores de la época, sino principalmente intelectuales de mediados del siglo veinte, como los eminentes economistas Jorge Ahumada (1958) y Aníbal Pinto Santa Cruz (1973), entre otros.

Con relación a la inflexión de término de la expansión de este ciclo, la hemos identificado con el año 1919. Sin embargo, antes es preciso hacer una referencia a la crisis de 1914, que fue particularmente dura para la industria de este fertilizante. En ese momento el nitrato de soda ya había perdido la competencia con los otros fertilizantes, especialmente con salitre sintético inventado en Alemania. Según Couyoumdjian, "el salitre estaba en desventaja en cuanto a costos; el costo del nitrato sintético por kilo de nitrógeno fluctuaba entre 5,76d y 8,64d, mientras que el costo equivalente del salitre oscilaba entre 10,2d y 13,2d" (1986:15). Sin embargo, como sería Alemania y su industria la principal afectada por la Gran Guerra iniciada ese año, la economía salitrera tendría un segundo aire para volver a crecer gracias a la demanda de nitrato refinado para fines bélicos.

Ninguna crisis anterior, por mucho que se haya anunciado o temido, tuvo igual alcance. Solamente después de resuelta de modo tan violento la huelga obrera de diciembre de 1907, se vio un abandono de la región salitrera a similar escala. En el segundo semestre de 1914 se inauguraron las ollas de pobres y los galpones para cesantes. Incluso debieron repatriarse obreros cesantes que habían llegado en enganches desde Bolivia ${ }^{1}$.

Sin embargo, la economía del salitre se recuperó a mediados de 1915 y seguirá esa tendencia hasta 1918 (Gráfico 3).

Como hemos afirmado fue la Guerra Mundial la que sacó a la industria salitrera de la crisis, pues en julio de 1915 la demanda de nitrato hizo cambiar todo el escenario de pobreza para volver a recibir enganches de trabajadores.

Un hecho relevante se constató con esta crisis, los empresarios no pudieron coludirse como lo venían haciendo desde 1884, mediante combinaciones salitreras, cerrando con ello el periodo del monopolio del nitrato chileno. Ya no estaría en sus manos el control de toda la industria como exitosamente lo habían hecho el Nitrate Permanent Comittee y la Asociación Salitrera de Propaganda hasta 1910. ¿Fue la menor presencia británica hacia 1914 en esta industria la que impidió la combinación?, ¿fue el conflicto entre nacionalidades de los salitreros, generado por la propia Guerra Mundial, como alemanes e ingleses, que estuvieron unidos desde la década de 1870 por los beneficios del salitre, lo que impidió la combinación?

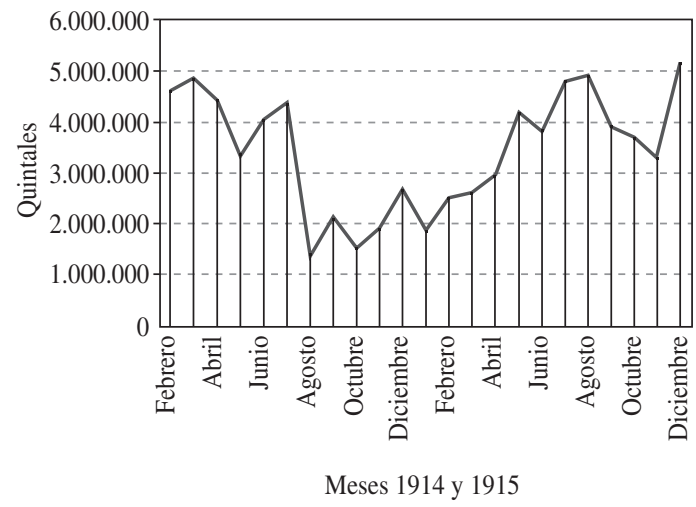

Gráfico 3. Exportaciones mensuales de salitre, años 1914-1915, en quintales.

Fuente: Archivo Histórico Nacional. Fondo del Salitre, Vol. 1103. 
Si comparamos las exportaciones de salitre del año 1914 y las del año 1915 (Gráfico 3) vemos que tienen un comportamiento como en espejo, pues en 1914 fue el segundo semestre el tiene una brusca caída (a partir de julio) mientras en 1915 el segundo semestre fue de recuperación. En rigor, entonces, la crisis solo fue de un año: entre julio de 1914 y junio de 1915, después se viviría un auge que duraría hasta el final de la Gran Guerra. Los años que continuaron y mientras duró la guerra fueron de auge. En la región salitrera hubo un impacto negativo en los cantones del Toco y de Taltal por la presencia de oficinas salitreras con capitales alemanes, las que se vieron compelidas a cerrar sus instalaciones.

Sin embargo, concluida la guerra y desaparecido el mercado de nitrato refinado para fines bélicos, la industria salitrera de Chile volvió a la realidad previa al conflicto: volver a competir en un mercado de fertilizantes, donde los costos del salitre chileno cada vez eran más altos y la competencia más dura. La crisis era inminente y con notoria fuerza.

En 1918 se edita un interesante libro titulado El salitre ante sus competidores, Soc. Imp. Lit. Universo, Santiago, donde su anónimo autor afirma con cierta ironía que "El Sr. Bertrand, en su propósito de mantener a sus juicios siniestros para el porvenir del salitre -y en esto lo han acompañado algunas entidades salitreras y hombres de reconocida situación-, ha llegado a suponer que Alemania como otros países europeos puedan llegar a gravar con derechos de importación a nuestro salitre, como un medio de amparar los enormes capitales invertidos durante la guerra en crear las fábricas de sustancias azoadas. Tal suposición, desprovista en nuestro concepto de todo fundamento, no debiera de ningún modo ser sustentada por el Sr. Bertrand, puesto que si esas fábricas producen tan económicamente, como lo ha aseverado en diferentes ocasiones, teniendo a la vista cálculos y datos bien documentados, no necesitarían de tal recurso para vivir y hacer prósperos sus negocios. Hay, pues, en esto cierta manifiesta contradicción" (1918:15). Efectivamente, Alemania no gravó al nitrato chileno, pero porque ya no requirió más de él, es decir, Bertrand quedó corto en su predicción.

Fue 1918, por lo anteriormente señalado, el último año que la economía salitrera estuvo en expansión efectiva. En rigor fue el año salitrero 1917/18. Si observamos las exportaciones de esta industria entre los años salitreros (de julio a junio) entre
1916/17 y 1924/5, según registros de la Asociación de Productores de Salitre (Cuadro 1 y Gráfico 4), vemos que la economía salitrera -a diferencia de la crisis de 1914- no volverá a recuperarse.

La tardía alarma de emergencia que se prendió en 1926 con la Semana del Salitre, realizada en Santiago en abril, solo sirvió para elaborar una explicación del desastre que ya había acontecido. El gerente de la Asociación de Productores de Salitre, en la mencionada Semana del Salitre, Alfredo Santander, nos relata que "el Fisco, vinculado en sus intereses al desarrollo y prosperidad de esta industria resolvió, desde el primer momento, adoptar una política salitrera, la idea de la unión de todos los industriales dentro de ciertas normas que eliminasen para siempre la especulación y diesen estabilidad y fijeza al comercio salitrero. $\mathrm{La}$ guerra mundial retardó, sin duda, la realización de

Cuadro 1. Exportación de nitrato chileno según año salitrero de julio a junio entre 1916/17 y 1924/25 (en quintales)

\begin{tabular}{lc}
\hline Años & Totales \\
\hline $1916 / 17$ & 62.249 .529 \\
$1917 / 18$ & 63.325 .382 \\
$1918 / 19$ & 39.007 .083 \\
$1919 / 20$ & 47.977 .583 \\
$1920 / 21$ & 44.581 .079 \\
$1921 / 22$ & 13.339 .948 \\
$1922 / 23$ & 21.032 .697 \\
$1923 / 24$ & 21.746 .994 \\
$1924 / 25$ & 25.659 .131 \\
\hline
\end{tabular}

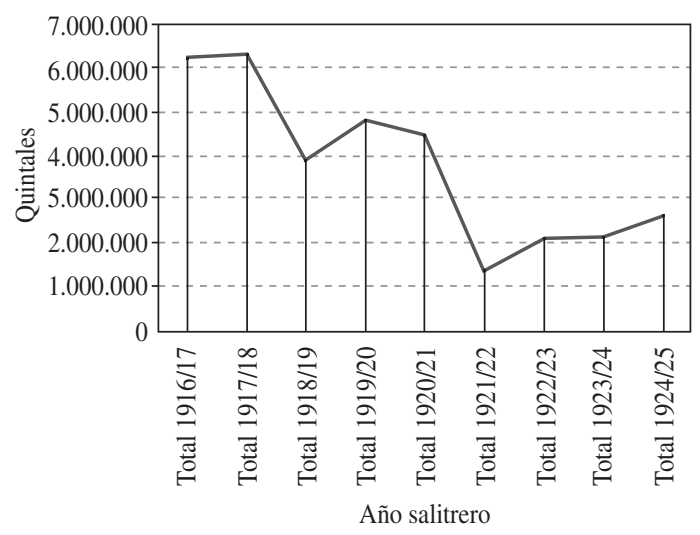

Gráfico 4. Exportación de nitrato chileno según año salitrero de julio a junio entre 1916/17 y 1924/25 (en quintales).

Fuente: Archivo Histórico Nacional. Fondo del Salitre, Vol. 1103. 
los propósitos que el gobierno de Chile y la gran mayoría de los productores tenían respecto de la forma de hacer las ventas" (1926:51). Es decir, la demanda generada por la guerra mundial postergaron las recomendaciones propuestas por Bertrand en 1911. Los industriales y el Estado-Nación chileno vieron que la gran amenaza llamada salitre sintético desaparecía de escena y aprovecharon el momento, sin pensar en mejorar las tecnologías y en cambiar la política salitrera.

Este autor también nos recuerda que el primer proyecto sobre organización de la industria salitrera fue en 1916, pero "diversos proyectos ocuparon la atención y discusión del Poder Legislativo hasta que, a principios de 1919, los industriales salitreros echaron, por acuerdo mutuo, las base de lo que es hoy la Asociación de Productores de Salitre de Chile" (1926:52). No solo sería una medida tardía sino insuficiente para el problema que se enfrentó en la década de 1920.

Jorge Vidal, quien fuera presidente de la Asociación de Productores de Salitre de Chile, afirmaba el 30 de julio de 1922: "La industria salitrera ha sido hasta ahora el ave doméstica en la cual se ha ejercitado el aparato del impuesto inflexible... Pero ha llegado el momento en que por mansa y bien dispuesta que esté la gallina, las fuerzas la abandonan y, si se quiere que viva, hay que reducirle la tarea" (1953:94). Efectivamente, la gallina siguió viva incluso más allá de la gran crisis internacional de 1929 , pero ya no era la misma del ciclo de expansión.

El problema no fue exclusivo de los empresarios, volvieron en todo el territorio salitrero los albergues para los cesantes y el embarque de los pampinos de retorno a sus lugares de origen, sea el sur de Chile, Cochabamba, Arequipa, etc. ${ }^{2}$ Todos vivían el mismo drama, siempre con la esperanza de retornar a la pampa como aconteció en 1915.

Un cambio interesante se constató en el periodo de la Primera Guerra Mundial y que se logró enmascarar: la pérdida de importancia económica y política de Tarapacá como la principal región productora y exportadora de nitrato chileno. En Tarapacá estaba localizada la Asociación Salitrera de Propaganda desde marzo de 1894, principal organización empresarial de salitreros en Chile, pero terminada la Guerra fue reemplazada por la Asociación de Productores de Salitre de Chile, cuya sede se ubicó en Valparaíso en enero de 1919. Paulatinamente habían abandonado la provincia las principales compañías salitreras, especialmente inglesas. Algunas comenzaron a trasladarse al sur del río Loa, al cantón central o Bolivia. Fueron los casos de salitreros que se iniciaron en Tarapacá como Eduardo Cavellero, Pedro Perfetti, Pascual Baburizza, entre otros.

Antofagasta fue la región salitrera que continuó a Tarapacá en el liderato de la exportación de nitrato chileno desde 1909 en adelante (Gráfico 5). Ni siquiera en los años de auge durante la Primera Guerra Tarapacá pudo alcanzar la producción unida de los cantones del Toco, Central, Aguas Blancas y Taltal.

Quizás la importancia de Antofagasta no fue destacada en el periodo porque disfrutó, al igual que Tarapacá, el auge económico de la Primera Guerra Mundial, y también sufrieron juntas la crisis a partir de 1919 (Gráfico 6). La diferencia más notoria entre ambas regiones se comenzó a percibir en la década

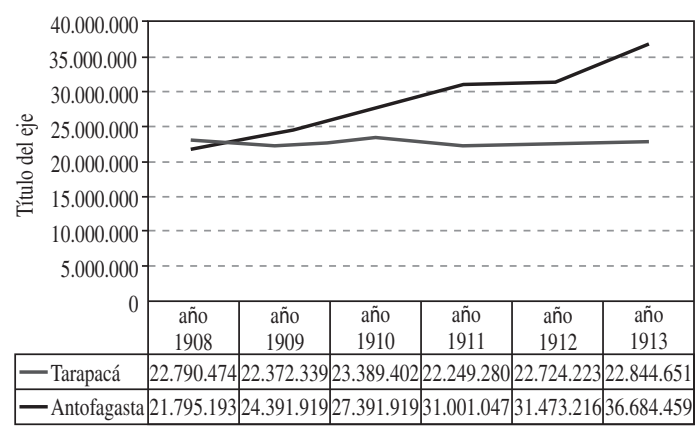

Gráfico 5. Eportación de salitre comparando regiones de Tarapacá y Antofagasta.

Fuente: Archivo Nacional, Asociación Salitrera de Propaganda Vol. 1103; Anuario Estadístico de Chile.

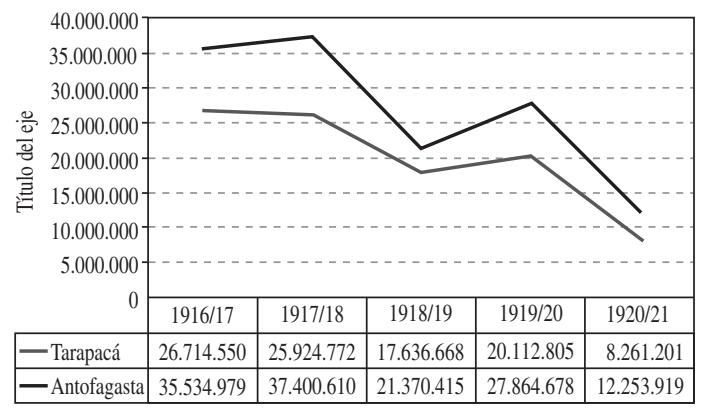

Gráfico 6. Eportación de salitre comparando regiones de Tarapacá y Antofagasta.

Fuente: Archivo Nacional, Asociación Salitrera de Propaganda Vol. 1103; Anuario Estadístico de Chile. 
de 1920, cuando el sistema de lixiviación de salitre Guggenheim comenzó a implementarse con éxito en las oficinas Pedro de Valdivia y María Elena, mientras al norte del río Loa se continuaría trabajando con un Shanks modificado hasta que cerró la última de sus grandes salitreras, Victoria, en 1978.

\section{Consideraciones finales}

Establecer los momentos de inflexión de inicio y término de un fenómeno social, que se desarrolló en un determinado territorio, no tiene por objetivo solamente enmarcarlo entre dos fechas para establecer su duración, sino destacar su importancia con ese territorio. Sumado a lo anterior, los momentos de inflexión de territorios aislados suelen establecerse según su relación con los centros económicos y políticos, especialmente con el Estado-Nación.

Para el caso del ciclo del salitre, que se desarrolló en el desierto de Atacama, en las provincias de Tarapacá y Antofagasta, en Chile su expansión económica la historiografía la enmarca entre 1880 y 1930, mientras en Perú solo es relevante el periodo que coincide con el gobierno de Manuel Pardo (1872-1976) y su política salitrera. La complejidad de este fenómeno es que se desenvolvió parcialmente en un territorio, la provincia de Tarapacá, que formó parte de dos Estados nacionales distintos: Perú y Chile, pero principalmente porque la perspectiva que prevaleció para analizar a esta industria ha provenido desde la escala nacional.

La perspectiva Estado-céntrica que establece la importancia de una industria regional suele, siguiendo a Llopis (2007), una mutilación del objeto de estudio. En este caso vemos un ciclo salitrero peruano y otro chileno, en referencia a una misma región: Tarapacá. Los ingresos del salitre entre 1872 y 1878 fueron fundamentales para el Perú, como lo destacan Bonilla (1984), Miller y Greenhill (2010), Contreras (2011), Neojevich y Vento (2009), entre otros; en cambio, para la historiografía chilena el ciclo de expansión del salitre se inicia en 1880 , haciéndolo coincidir con la administración chilena.

La perspectiva a escala nacional ha llevado a entender a la región salitrera como un enclave económico y social, al ser vista como económicamente estratégica para el país, pero territorialmente marginal, aislada y despoblada, sin comprender las dinámicas sociológicas y culturales propias de la sociedad que se fue forjando en ese territorio con relación a la industria del nitrato, desde la fundación de los primeros campamentos, pueblos y puertos salitreros hasta la actualidad, donde todavía es posible identificar organizaciones de pampinos en ciudades como Arica, Iquique, Tocopilla y Antofagasta. Para estos pampinos y sus antecesores el ciclo del salitre es uno solo, desde las primeras hasta las últimas exportaciones de nitrato de soda. Aquí hemos revisado un periodo más breve que dice relación con el momento de expansión económica de esta industria.

\section{Referencias Citadas}

Chernilo, Daniel

2006 "Social Theory's Methodological Nationalism: Myth and Reality". European Journal of Social Theory, 9 (1), 5-22.

Bermúdez, O.

1963 Historia del salitre desde sus orígenes hasta la guerra del Pacífico. Ediciones Universidad de Chile, Santiago.

Bertrand, A.

1912 Tributación salitrera. Estudio acerca de su mejor adaptación al mercado postbélico. Imprenta y Litografía Universo, Santiago.

Bertrand, A.

1918 Política salitrera. Bases de un programa de defensa del salitre. Imprenta y Litografía Inglesa R. Magowan, Valparaíso.

Billinghurst, G.

1875 Rápida ojeada sobre la Cuestión Salitre. Imprenta del Mercurio, Valparaíso.
Billinghurst, G.

1889 Los capitales salitreros de Tarapacá. Imprenta El Progreso, Santiago.

Bonilla, $\mathrm{H}$.

1984 Guano y Burguesía en el Perú. Instituto de Estudios Peruanos IEP, Lima.

Bonilla, $\mathrm{H}$

1985 "La crisis de 1872". Documentos de Trabajo No 64, junio, PUCP, Lima.

Bravo, J.A.

1990 The peruvian expropiation of the Tarapaca nitrate industry, 1875-1879. Thesis for the Degree of Doctor of Philosophy, Departament of History, Mc Gill University, Montreal.

Collier, S. y Sater, W.

1996 Historia de Chile 1808-1994. Cambridge University Press. Contreras, C., M.

2012 La economía pública en el Perú después del guano y del salitre. Instituto de estudios Peruanos, IEP, Lima. 
Couyoumdjian, J.R.

1986 Chile y la Gran Bretaña durante la primera guerra mundial y la posguerra 1914 - 1921. Editorial Andrés Bello y Ediciones de la Pontificia Universidad Católica de Chile, Santiago.

Crozier, R.

1997 "El salitre hasta la Guerra del Pacífico. Una revisión". Historia Vol. 30, PUC, 53-126.

Díaz Ossa, B.

1926 "La industria salitrera chilena". En: Urzúa et al. Semana del salitre celebrada en Santiago de Chile, abril 1926. Imprenta y Litografía La Ilustración, Santiago, 141-170.

Jessop, R.

2004 "La economía política de la escala y la construcción de las regiones transfronterizas". EURE, Vol. XXIX, № 89 , 25-41, PUC, Santiago.

Grimson, A.

2005 "Fronteras, Estados e identificaciones en el Cono Sur". En: Mato, D. Cultura, política y sociedad. Perspectivas latinoamericanas. CLACSO, 127-142.

Grosfoguel, R.

2006 "Del final del sistema-mundo capitalista hacia un nuevo sistema-histórico alternativo: la utopística de Immanuel Wallerstein". Nómadas $\mathrm{N}^{\circ}$ 25, 44-52, Universidad Central, Colombia.

Hernández, R.

1930 El salitre. Fisher Hnos., Valparaíso.

Hunt, S.

2011 La formación de la economía peruana. Distribución y crecimiento en la historia del Perú y América Latina. IEP, PUCP, Lima.

Llopis, R.

2007 “El 'Nacionalismo metodológico' como obstáculo en la investigación sociológica sobre migraciones internacionales". Empiria. Revista de metodología de Ciencias Sociales, 13, 101-117.

Marichal, C.

2009 "La crisis mundial de 1873 y su impacto en América Latina". ISTOR Revista de Historia Internacional, CIDE, $\mathrm{N}^{\circ}$ 36, año IX, 21-47.

Mathew, W.

2009 La firma inglesa Gibbs y el monopolio del guano en el Perú, Lima, Instituto de Estudios Peruanos, 2009.

Masso, E.

2013 "Superando el nacionalismo metodológico: Comunidades cosmopolitas de interacción en el barrio de Lavapiés". Migraciones Internacionales, Vol. 7, $\mathrm{N}^{\circ}$ 2, julio-diciembre, 71-100.

Morcillo, A.

2011 "El debate entre transnacionalismo y nacionalismo metodológico como marco teórico para la comprensión del papel del empleo en la gobernabilidad de la inmigración en España”. Papers, $N^{\circ}$ 96/3, 757-780.
Miller, R. y Greenhill, R.

2011 "El gobierno peruano y el comercio del salitre, 1873-

1879”. En: Miller, R. Empresas británicas, economía y política en Perú 1850-1934. Instituto de Estudios Peruanos IEP, 33-67, Lima.

Mücke, U.

2004. "Elecciones y participación política en el Perú del siglo XIX: la campaña presidencial de 1871-72". Investigaciones Sociales $\mathrm{N}^{\circ}$ 12, Año VIII, 133-166.

Noejovich, H.O. y Vento, A.

2009 Guano, salitre y finanzas públicas: el Pacífico en el siglo XIX. Documento de Trabajo No 273, PUCP, Lima.

O'Brien, T.

1982 The nitrate industry and Chile's crucial transition: 1870-1891. New York University Press.

Ravest, M.

2008 "La Casa Gibbs y el monopolio salitrero peruano: 1876-1878”. Historia $N^{\circ} 41$, vol. I, enero-junio, 63-77.

Reyes Flores, A.

1979 "Relaciones internacionales en el Pacífico Sur, ensayo de interpretación: 1873-1879”. En: La Guerra del Pacífico, Universidad Mayor de San Marcos, Vol. I, 39-96, Lima.

Ruz, R., Díaz, A., Galdames, L.

2008 Población andina de las provincias de Arica y Tarapacá. El censo de 1866. Ediciones de la Universidad de Tarapacá, Arica.

Santander, A.

1926 "Asociación de productores de salitre de Chile. Su organización; sus propósitos; sus fines". En: Comisión Consultiva de Salitres Informe que la Comisión Consultiva de Salitres presenta al Señor Ministro de Hacienda. Imprenta Nacional, Santiago, 49-78.

S/autor

1981 "El salitre ante sus competidores". Soc. Imp. Lit. Universo, Santiago.

Semper, E. y E. Michels

1908 La industria del salitre en Chile. Imprenta, Litografía y Encuadernación Barcelona, Santiago.

Vidal, J.

1953 La tragedia del salitre. Imprenta el imparcial, Santiago.

\section{Archivos y fuentes primarias}

Archivo Nacional, Asociación Salitrera de Propaganda

Archivo de Intendencia de Tarapacá, Chile (AIT)

Archivo Histórico Nacional. Fondo Prefectura de Tarapacá (AHN)

Archivo General de la Nación, Perú (AGN)

Archivo Riva Agüero. Colección Denegri.

Archivo Municipal de Lima.

Periódico La Libertad Electoral.

Periódico El Nacional.

Periódico La Patria.

Periódico El Nacional.

Periódico El Peruano.

\section{Notas}

1 A modo de ejemplo, vemos una carta del cónsul de Bolivia en Iquique, Arístides Moreno, dirigida al Intendente de Tarapacá, Agustín Arrieta, a saber: Consulado de Bolivia, Iquique $\mathrm{N}^{\circ} 118$
Iquique, octubre 14 de 1914.

Señor Intendente:

Tengo el honor de dar respuesta á su atenta nota $\mathrm{N}^{\circ} 1494$, de fecha 8 del corriente, por la que se dignó U.S. poner en mi conocimiento que esa Intendencia ha sido autorizada por 
el Supremo Gobierno para repatriar á los obreros bolivianos que han quedado cesantes por la paralización de las faenas salitreras.

U.S. me insinúa que ponga este hecho en conocimiento de mis connacionales, haciéndoles presente que la situación aquí, cada día les será más difícil y que el Supremo Gobierno no podrá seguir alimentando por más tiempo, á los obreros cesantes.

Siguiendo la práctica anteriormente establecida, he continuado extendiendo algunas papeletas de solicitud de pasajes, pero ellas, según lo afirman los interesados, parece que no han sido atendidas por esa Intendencia ni tampoco por las agencias de los Vapores "Chancay" y "Salvador", (que eran los que llevaban trabajadores bolivianos a Arica), asegurando no tener orden de U.S.

En su mencionado oficio se digna U.S. solicitarme datos sobre el número de obreros y sus familias que existen en esta ciudad. A este respecto siento decir a U.S. que carezco de tales datos, los cuales, por otra parte, no pueden menos de variar cada instante. Hoy existían 29 bolivianos en el Hipódromo. Agradeciendo á U.S. su atenta comunicación, tengo la honra de reiterarle mis más sinceros respetos.

\section{A. Moreno}

Cónsul de Bolivia, Decano del cuerpo consular. Fuente: Archivo de Intendencia de Tarapacá, Libro Consulados, fojas 266, ubicación ITAR 834, años 1911-1915.

2 A modo de ejemplo esta noticia del Nacional de Iquique del 20 de enero de 1919, titulado "Obreros al sur": "Por vapor Fresia se dirijeron ayer 250 obreros con sus familias, que han quedado cesantes con motivo de la crisis salitrera. En el Taltal que llega el miércoles también se remitirán otros tantos obreros. Volvemos a advertir que solo se dá pasaje al sur a los obreros que comprueben que vienen de oficinas que han cesado sus labores". 
